\section{PO 8592 WHY, WHEN AND WHERE DO NEWBORNS NOT ONLY GET SICK BUT ALSO DIE IN SÃO TOMÉ AND PRÍNCIPE? A CASE-CONTROL STUDY}

${ }^{1}$ Alexandra Vasconcelos*, ${ }^{2}$ Swasilanne Sousa, ${ }^{2}$ Nelson Bandeira, ${ }^{3}$ João L Baptista, ${ }^{4}$ Maria Do Céu Machado, 'Filomena Pereira. 'Global Health and Tropical Medicine/Instituto de Higiene e Medicina Tropical, Universidade Nova de Lisboa, Portugal; ${ }^{2}$ Hospital Dr. Ayres de Menezes, São Tomé e Príncipe; ${ }^{3}$ Faculdade Ciências da Saúde, Universidade da Beira Interior, Covilhã, Portugal; ${ }^{4}$ Faculdade de Medicina de Lisboa, Universidade de Lisboa, Lisbon, Portugal

\subsection{6/bmjgh-2019-EDC.157}

Background Neonatal deaths in São Tomé and Príncipe account for about $43 \%$ of all under-5 deaths, but there are major gaps in understanding this. The objective of this study was to identify ante-, intra-, and post-partum risk factors and to analyse characteristics of neonatal morbimortality.

Methods Stillbirths and sick newborns (NB) with $\geq 32$ week's gestation or $\geq 1500 \mathrm{~g}$ were eligible cases, while controls were healthy newborns $\geq 28$ days. The study is still underway, and more data will become available. Results presented here are from a questionnaire applied by the investigator, and from medical records of mothers and newborns. Point-of-care tests were used to screen for syphilis, HIV and Hepatitis B. The SPSS 23 statistical programme was used for data analysis. Informed consent was obtained from every mother included in the study.

Results Out of 675 mothers, 132 were enrolled corresponding to 134 newborns (2 twins), while 35 cases and 41 newborn controles were followed up. Pregnant women's medium age was 26 years, $23 \%$ being adolescents and 23\% without antenatal care. Primary education was attended by $57 \%$, secondary by $63 \%, 7 \%$ never attended school. Syphilis - 1/96 reactive test; HIV or malaria not detected; 3 infected with HBV. Newborn morbimortality: 10 preterm, 12 birth asphyxia, 30 with risk of neonatal and 8 with invasive infections, 8 foetal growth restriction, 5 microcephaly, 4 minor congenital anomalies and 1 death in the first 24 hours of life (congenital lung anomaly). Low birth weight $(<2500 \mathrm{~g})$, meconium and caesarean section were statistically significant with respect to morbidity $(\mathrm{p}<0.05)$. No deaths were verified in the 76 babies followed up.

Conclusion In this study, newborn morbidity was high (59\%). More conclusions will be drawn when a higher number of participants is included and analysed. As a result, at the completion of this study, we hope to be able to design an intervention algorithm in order to achieve peri-neonatal morbimortality reduction.

\section{PO 8594 VALIDATION OF A VERBAL AUTOPSY QUESTIONNAIRE APPLIED TO A CASE-CONTROL STUDY OF CEREBRAL MALARIA IN BANDIAGARA AND BAMAKO, MALI}

Karim Traore*, Drissy Coulibaly, Abdoulaye K Kone, Ali Thera, Boureima Guindo, Bourama M Tangara, Aichatou A Diawara, Ogobara K Doumbo†, Mahamadou A Thera. MRTC/ FMOS, University of Sciences, Techniques and Technologies of Bamako, Bamako, Mali

\subsection{6/bmjgh-2019-EDC.158}

Background Malaria remains a leading cause of child mortality in sub-Saharan Africa. Severe malaria is the main pathway to death caused by malaria. A study of factors associated with protection against severe malaria is best achieved using a casecontrol design. However, these studies are affected by the quality and appropriateness of the choice of controls that may introduce important bias in study design and definitively alter the conclusions from the studies. It is of paramount importance that the presence or absence of an episode of severe malaria in the history of the controls is ascertained. In settings with reliable medical data this is easily tracked back from hospital records. In settings like Bandiagara, with weak health care systems, a dedicated approach is needed to track history of severe malaria. We used an adapted verbal autopsy questionnaire to identify presence of cerebral malaria in the history of controls in a case-control study of severe malaria in Bandiagara.

We have assessed the sensitivity, specificity and predictive positive and negative values of the questionnaire.

Methods From January through August 2018 we enrolled 450 children with or without known confirmed cerebral malaria selected at health care centers in Bandiagara and Bamako, with archived medical records. Parents of enrolled children were identified, located and interviewed using the adapted verbal autopsy questionnaire. Interviewers were not aware of children's diagnosis. We compared the diagnosis derived from the verbal autopsy questionnaire to the confirmed biological diagnosis from the medical records.

Results We will present full results on the sensitivity, specificity, the predictive positive and negative values of the verbal questionnaire, and the Kappa coefficient of agreement.

Conclusion A good verbal questionnaire will enhance the quality of the choice of controls in case-control studies of severe malaria and overall will improve the quality and relevance of inferences from such studies.

\section{PO 8595 FACTORS ASSOCIATED WITH VIROLOGIC FAILURE AMONG WOMEN WITH PRIOR EXPOSURE TO ANTIRETROVIRAL DRUGS FOR PMTCT, KISUMU, KENYA}

${ }^{1,3}$ Francis Angira*, 'Eucabeth Awuonda, 'Jacinter Oruko, 'yaro Boaz, ${ }^{2}$ Elijah Asadhi, ${ }^{1}$ George Olilo. 'Kenya Medical Research Institute-Center for Global Health Research, Kenya; ${ }^{2}$ United Nations Children's Fund (UNICEF), Nairobi, Kenya; ${ }^{3}$ East African Consortium for Clinical Research, Nairobi, Kenya

\subsection{6/bmjgh-2019-EDC.159}

Background Use of antiretroviral drugs (ARVs) for a discrete period for Preventing Mother-to-Child HIV transmission (PMTCT) only may be compared to Structured Treatment Interruption, which has been associated with virologic failure (VF). We sought to determine factors associated with VF among women on Antiretroviral Therapy (ART) but with prior exposure to short-term ARVs for PMTCT.

Methods HIV-infected women presenting for ART initiation in three HIV care clinics in Kisumu County, Kenya were enrolled in the KiBS follow-up study (2010-2013) if they had previously received triple ARVs for PMTCT (Group 1) or shortcourse ARVs for PMTCT (Group 2) or were ARVs-naïve (Group 3). First-line ART was provided as per 2010 WHO treatment guidelines and viral load (VL) tests were conducted every six months for 24 months. VF was defined as any confirmed VL value $\geq 400 \mathrm{copies} / \mathrm{ml}$ after 6 months of ART initiation. Frequencies and proportions were used in the descriptive analysis while Pearson's Chi-square/Fisher's exact test was used to determine the association between VF and eight independent variables. Univariate and Multivariate Coxproportional regression model was fitted to investigate factors associated with VF. 